\title{
Desempenho de Trichogramma pretiosum Riley, 1879 (Hymenoptera: Trichogrammatidae), submetido a inseticidas e fungicidas em dois hospedeiros ${ }^{1}$
}

Vinícius Pereira dos Santos ${ }^{2}$, Dirceu Pratissoli², João Paulo Pereira Paes ${ }^{3}$, Débora Ferreira Melo Fragoso*2, José Romário de Carvalho ${ }^{2}$

10.1590/0034-737X201663050009

\section{RESUMO}

Este trabalho teve como objetivo avaliar a influência de diferentes inseticidas e fungicidas, sobre Trichogramma pretiosum Riley, 1879 (Hymenoptera: Trichogrammatidae), em diferentes hospedeiros. Os testes foram realizados com adultos de T. pretiosum nos hospedeiros Anagasta kuehniella (Zeller, 1879) (Lepidoptera: Pyralidae) e Helicoverpa zea (Boddie, 1850) (Lepidoptera: Noctuidae). Os resultados mostraram que os agrotóxicos estudados tiveram efeitos diferentes sobre T. pretiosum, em função do hospedeiro. Os inseticidas IMIDACLORIPRIDO + BETA-CIFLUTRINAe METHOMYL foram os mais agressivos, reduzindo o parasitismo dos adultos de T. pretiosum em ambos os hospedeiros, enquanto, para os fungicidas, DIMETOMORPH + MANCOZEB e MANCOZEB foram os mais influentes. Diante dos resultados, concluise que o hospedeiro pode influenciar na ação seletiva dos inseticidas e fungicidas sobre parasitoides do gênero Trichogramma, visto que T. pretiosum foi mais susceptível ao inseticida METHOMYLe ao ACIBENZOLAR-S-METHYL, quando criado em A. kuehniella, e ao fungicida DIMETOMORPH + MANCOZEB, quando criado em H. zea. Não houve reduções expressivas dos percentuais de emergência dos descendentes, quando os fungicidas foram aplicados.

Palavras-chave: Parasitoide; Agrotóxicos; Manejo Fitossanitário; Hospedeiros alternativos.

\section{ABSTRACT}

\section{Performance of Trichogramma pretiosum Riley, 1879 (Hymenoptera: Trichogrammatidae) submitted to insecticides and fungicides in two hosts}

This study aimed to evaluate the influence of different insecticides and fungicides to Trichogramma pretiosum Riley, 1879 (Hymenoptera: Trichogrammatidae) in different hosts. The tests were performed on adults of T. pretiosum in host Anagasta kuehniella (Zeller, 1879) (Lepidoptera: Pyralidae) and Helicoverpa zea (Boddie, 1850) (Lepidoptera: Noctuidae). The results showed that the pesticides studied had different effects on T. pretiosum depending on the host. The insecticides IMIDACLORIPRIDO + BETA-CYFLUTHRIN and METHOMYL were the most aggressive reducing parasitism of adult $T$. pretiossum in both hosts, whereas for fungicides, DIMETOMORPH + MANCOZEB and MANCOZEB were the most influential. From the results it is concluded that the host can influence the selectivity tests of insecticides and fungicides on parasitoids of the genus Trichogramma, considering, T. pretiosum was more susceptible to the insecticide METHOMYL and ACIBENZOLAR-S-METHYL when created in A. kuehniella and the fungicide MANCOZEB + DIMETOMORPH created when $H$. zea. There were no significant reductions in emergency percentage of descendants when fungicides were applied.

Key words: Parasitoid; Pesticides; Plant management; Alternative hosts.

\footnotetext{
Submetido em 06/07/2015 e aprovado em 29/10/2015.

${ }^{1}$ Este trabalho é parte da dissertação de mestrado do primeiro autor.

${ }^{2}$ Universidade Federal do Espírito Santo, Centro de Ciências Agrárias, Departamento de Produção Vegetal, Alegre, Espírito Santo, Brasil. agronomovinicius@gmail.com; dirceu.pratissoli@gmail.com; debmelo@gmail.com; jromario_carvalho@hotmail.com

${ }^{3}$ Universidade Estadual Paulista "Júlio de Mesquita Filho", Departamento de Proteção Vegetal, Botucatu, São Paulo, Brasil. joaopauloppaes@hotmail.com

*Autor para correspondência: debmelo@gmail.com
} 


\section{INTRODUÇÃO}

A busca pela redução de perdas nas lavouras de tomate tem levado agricultores ao uso massivo e indiscriminado de agrotóxicos, por causa dos problemas fitossanitários (Carvalho et al., 2012). Dentre esses, a elevada susceptibilidade da cultura a doenças e o frequente ataque de insetos-praga são os principais entraves da cadeia produtiva, o que classifica o tomateiro como cultura de alto risco (Luz et al., 2007).

Visando à redução do uso de inseticidas, a utilização de outros métodos de controle ou, sua associação com inseticidas, torna-se indispensável para o manejo integrado e, consequentemente, para a redução dos insetos-praga (Alves et al., 2007). Um método de manejo bastante estudado e utilizado é o controle biológico, utilizando-se parasitoides do gênero Trichogramma (Almeida et al., 2010). A utilização desses agentes em associação com agrotóxicos pode ter sua eficiência comprometida pela ação desses produtos sobre os parasitoides. Diante disso, torna-se necessária a realização de testes que verifiquem o efeito dos agrotóxicos sobre esses organismos, gerando informações que possam auxiliar na tomada de decisão em programas de Manejo Integrado de Pragas (Pratissoli et al., 2002; Stefanello Júnior et al., 2012; Carvalho, et al., 2012).

Para auxiliar nesses testes de seletividade e susceptibilidade, a International Organization for Biological Control of Noxious Plants and Animals/ West Palaearctic Region Section (IOBC/WPRS) desenvolveu um protocolo padrão, que visa a avaliar o grau de toxicidade e o impacto que os agrotóxicos causam sobre Trichogramma (Hassan et al., 1998; Pratissoli et al., 2009).

Em função disso, este estudo teve como objetivo avaliar a influência de inseticidas e fungicidas, pertencentes a cada classe toxicológica, sobre Trichogramma pretiosum Riley, 1879 (Hymenoptera: Trichogrammatidae), nos hospedeiros alternativos Anagasta kuehniella (Zeller, 1879) (Lepidoptera: Pyralidae) e Helicoverpa zea (Boddie, 1850) (Lepidoptera: Noctuidae), bem como classificar os agrotóxicos quanto ao impacto sobre o parasitoide.

\section{MATERIAL E MÉTODOS}

Os experimentos de seletividade foram realizados no setor de Entomologia, do Núcleo de Desenvolvimento Científico e Tecnológico em Manejo Fitossanitário (NUDEMAFI), do Centro de Ciências Agrárias da Universidade Federal do Espírito Santo (CCA-UFES), em Alegre, ES. Todos os experimentos foram realizados em câmara climatizada, regulada com as seguintes características: temperatura a $25 \pm 1{ }^{\circ} \mathrm{C}$, umidade relativa (UR) $70 \pm 10 \%$ e fotofase de 14 horas.

\section{Criação e manutenção dos hospedeiros alternativos}

Para a criação de $H$. zea, as lagartas foram coletadas em lavouras de tomate da área experimental do CCA-UFES, localizado no município de Alegre, ES.

A fase imatura da praga foi criada em frutos de tomate (Solanum lycopersicum L.), até atingir a fase adulta ( $25 \pm 1$ ${ }^{\circ} \mathrm{C}$, UR de $70 \pm 10 \%$ e fotofase de $14 \mathrm{~h}$ ). Após a emergência, os adultos foram mantidos em gaiolas de PVC (20 cm de diâmetro x $25 \mathrm{~cm}$ de altura) revestidas internamente com folha de papel branco, sendo a extremidade superior fechada com tecido do tipo "voil" e, a inferior, com uma placa quadrada de isopor ( $25 \mathrm{~cm}$ de lado x $3 \mathrm{~cm}$ de espessura).

Diariamente, foi oferecida uma solução de mel a $10 \%$ $(\mathrm{m} / \mathrm{v})$ como substrato alimentar, por meio de algodão embebido na solução em frasco de vidro $(5 \mathrm{~mL})$. Os pedaços de tecido do tipo "voil", contendo as posturas, foram acondicionados em potes, que foram mantidos em condições controladas $\left(25 \pm 1^{\circ} \mathrm{C}, \mathrm{UR}\right.$ de $70 \pm 10 \%$ e foto fase de $\left.14 \mathrm{~h}\right)$, até a eclosão das lagartas.

As lagartas recém-eclodidas foram acondicionadas em tubos de vidro $(2,5 \mathrm{~cm}$ de diâmetro x $8,5 \mathrm{~cm}$ de altura), contendo dieta artificial de Greene et al. (1976) modificada por Parra (2001), para criação de Anticarsia gemmatalis (Hübner) (Lepidoptera: Noctuidae), sendo transferida 1 lagarta por tubo, que permaneceu até a fase de pupa.

As pupas coletadas nos tubos foram acondicionadas em gaiolas de acrílico até a emergência dos adultos e, após a emergência, estes foram colocados nas gaiolas de tubo PVC, com remoção do tecido tipo "voil", colocado para oviposição dos adultos, realizada diariamente, sendo os insetos transferidos para tubo PVC, com as mesmas condições citadas anteriormente.

Para A. kuehniella, a manutenção foi feita de acordo com a metodologia desenvolvida por Pratissoli et al. (2010a).

\section{Criação de e manutenção de Trichogramma pretiosum Riley, 1879 (Hymenoptera: Trichogrammatidae)}

Na manutenção e multiplicação do parasitoide $T$. pretiosum, empregou-se o hospedeiro alternativo $A$. kuehniella, cujos ovos foram aderidos às cartelas de cartolina azul celeste $(8 \times 2 \mathrm{~cm})$, utilizando-se goma arábica diluída a $20 \%(\mathrm{~m} / \mathrm{v})$. Esses ovos foram, posteriormente, submetidos à inviabilização sob lâmpada germicida por um tempo de 50 minutos, conforme descrito por Pratissoli et al. (2010a), sendo posteriormente expostos ao parasitismo por um período de 24 horas e mantidos em câmara climatizada, a $25 \pm 1{ }^{\circ} \mathrm{C}$, UR de $70 \pm 10 \%$ e fotofase de 14 horas. 


\section{Agrotóxicos testados}

Os produtos utilizados nos testes encontram-se listados na Tabela 1. A escolha dos produtos foi realizada por meio de sorteio ao acaso, levando-se em consideração somente sua separação por classe de uso (inseticida ou fungicida).

Os estudos de seletividade foram divididos em dois experimentos. No primeiro, foram avaliados os produtos pertencentes à classe dos inseticidas; no segundo, os produtos pertencentes à classe dos fungicidas.

Todos os agrotóxicos utilizados nos bioensaios são autorizados e registrados para utilização em lavouras de tomate, respeitando-se a legislação brasileira, Lei n ${ }^{\circ} 9.974$, de 06 de junho de 2000 (Agrofit, 2015).

\section{Bioensaio de seletividade}

Fêmeas recém-emergidas de T. pretiosum (idade $<24$ horas) foram individualizadas em tubos tipo Eppendorf (2,5 mL), com uma gotícula de mel no seu interior, para alimentação. Cartelas de cartolina azul celeste $(0,5 \times 3,0 \mathrm{~cm})$, com 20 ovos retirados da criação do hospedeiro $A$. kuehniella, ou do hospedeiro H. zea, colados por meio de goma arábica $(25 \% \mathrm{~m} / \mathrm{v})$, foram inviabilizadas por exposição à lâmpada germicida por aproximadamente 50 minutos.

As cartelas com os ovos foram imersas nas caldas dos produtos pertencentes aos Experimentos I ou II, por cinco segundos. No tratamento testemunha, utilizou-se somente água destilada como padrão. Em seguida, as cartelas foram colocadas sobre papel toalha, dentro de bandejas plásticas, em câmara de exaustão, durante uma hora, em temperatura ambiente, para eliminação do excesso de umidade da superfície dos ovos. As cartelas com os ovos tratados foram oferecidas ao parasitismo pelas fêmeas de T. pretiosum, previamente individualizadas nos tubos tipo Eppendorf (2,5 mL), durante 24 horas. Após esse período, as fêmeas foram eliminadas e as cartelas mantidas em tubos, para observação até a emergência dos parasitoides.
Os parâmetros observados foram: percentual de parasitismo, avaliado pela característica de escurecimento dos ovos (Pratissoli et al., 2007), e percentual de emergência. O número de indivíduos por ovo não foi avaliado, em virtude de haver uma diferença quanto ao tamanho dos ovos dos hospedeiros em estudo. Cada tratamento foi composto por 15 repetições, cada uma representada por uma cartela com 20 ovos do hospedeiro em estudo.

\section{Análise estatística}

O delineamento utilizado para o bioensaio de seletividade foi o inteiramente casualizado, em esquema fatorial 5 x 2 (tratamentos x hospedeiros), com 15 repetições cada, para cada experimento.

Os dados de parasitismo e emergência obtidos foram submetidos à análise de variância (ANOVA) e as médias comparadas pelo teste de Tukey, a 5\% de significância. A análise estatística foi realizada com o programa estatístico SISVAR versão 5.1 (Ferreira, 2003; 2011).

A redução do parasitismo ou da emergência dos parasitoides, para cada tratamento, foi determinada por comparação com a testemunha (água destilada) e calculada por meio da fórmula: RP = (1 - Rt/Rc) x 100, sendo RP a percentagem de redução do parasitismo ou de emergência; Rt o valor do parasitismo ou emergência média (a) para cada produto e Rc o parasitismo ou a emergência média (a) observado (a) para o tratamento testemunha (negativa) (Hassan et al., 2000). Com base nestas percentagens de redução, os produtos testados foram enquadrados em classes, de acordo com as recomendações da IOBC/WPRS (Hassan et al., 1998, 2000; Pratissoli et al., 2009) em que: 1 = inócuo $(<30 \%), 2=$ pouco prejudicial $(30-79 \%), 3=$ moderadamente prejudicial $(80-99 \%)$ e $4=$ prejudicial $(>$ 99\%).

\section{RESULTADOS E DISCUSSÃO}

A análise dos dados de parasitismos do Experimento I (inseticidas) mostrou significância da interação entre os

Tabela 1: Agrotóxicos utilizados no teste de seletividade sobre Trichogramma

\begin{tabular}{|c|c|c|c|}
\hline Nome técnico & [ ]i.a.* & Formulação & Classificação toxicológica \\
\hline \multicolumn{4}{|c|}{ Experimento I - Inseticidas } \\
\hline \multicolumn{2}{|c|}{ IMIDACLOPRIDO + BETA-CIFLUTRINA100+12,5 $\mathrm{g} \mathrm{L}^{-1}$} & SC & II - Altamente tóxico \\
\hline METHOMYL & $215 \mathrm{~g} \mathrm{~L}^{-1}$ & SL & I-Extremamente tóxica \\
\hline ACETAMIPRID & $200 \mathrm{~g} \mathrm{~kg}^{-1}$ & WP & III - Medianamente tóxico \\
\hline SPINOSAD & $480 \mathrm{~g} \mathrm{~L}^{-1}$ & SC & IV - Pouco tóxico \\
\hline \multicolumn{4}{|c|}{ Experimento II - Fungicidas } \\
\hline DIMETOMORPH + MANCOZEB & $90+600 \mathrm{~g} \mathrm{~kg}^{-1}$ & WP & II - Altamente tóxico \\
\hline ACIBENZOLAR-S-METHYL & $500 \mathrm{~g} \mathrm{~kg}^{-1}$ & WG & III - Medianamente tóxico \\
\hline MANCOZEB + OXICLORETO DE & BRE440+300 $\mathrm{g} \mathrm{kg}^{-1}$ & WP & IV - Pouco tóxico \\
\hline MANCOZEB & $800 \mathrm{~g} \mathrm{~kg}^{-1}$ & WP & I-Extremamente tóxica \\
\hline
\end{tabular}


fatores Tratamento (inseticidas) e Hospedeiro ( $\mathrm{F}=3,111$; glr $=107 ; p=0,0294)$ (Tabela 2). Ao realizar o desdobramento dos níveis do fator Inseticidas dentro dos níveis do fator Hospedeiro, verificou-se que o parasitismo de $T$. pretiosum foi influenciado de forma semelhante nos dois hospedeiros. Quando tratados com o inseticida SPINOSAD, T. pretiosum obteve as melhores taxas de parasitismo, assemelhando-se com o tratamento testemunha, para ambos os hospedeiros $(78,57$ e 85,67\% de parasitismo para os hospedeiros A. kuehniella e H. zea, respectivamente). O inseticida IMIDACLOPRIDO + BETACIFLUTRINA foi o que mais afetou o parasitismo, que, por sua vez, foi inferior a $30 \%$, para ambos hospedeiros. No desdobramento dos níveis do fator Hospedeiro, dentro dos níveis do fator Inseticidas, verificou-se uma redução significativa da taxa de parasitismo nos tratamentos com IMIDACLOPRIDO + BETA-CIFLUTRINA, quando o hospedeiro era A. kuehniella, e ACETAMIPRID, quando o hospedeiro era $H$. zea.

A taxa de redução do parasitismo em relação ao grau de parasitismo no tratamento testemunha, independentemente do hospedeiro, foi maior quando o parasitoide foi submetido ao inseticida IMIDACLOPRIDO + BETACIFLUTRINA, sendo superior a $80 \%$, quando o hospedeiro foi $A$. kuehniella, e superior a $60 \%$, quando o hospedeiro foi $H$. zea. Já as menores taxas foram observadas quando a aplicação foi com SPINOSAD, sendo que, em $A$. kuehniella, foi de apenas 4,57 vezes; no entanto, no hospedeiro $H$. zea, não houve redução e sim um parasitismo 6,14 vezes superior ao da testemunha (Tabela 2).

Com relação à classificação da IOBC, somente o tratamento SPINOSAD enquadrou-se na classe 1 (inócuo) para os hospedeiros, sendo que, para o hospedeiro H. zea, o parasitismo foi maior que o da testemunha específica $(85,67$ e $80,71 \%$, respectivamente) (Tabela 2 ). O tratamento METHOMYL apresentou classe 2 da IOBC, para ambos os hospedeiros. Já o tratamento ACETAMIPRID foi classe 1 para o hospedeiro A. kuehniella e classe 2 para o hospedeiro $H$. zea, enquanto o tratamento IMIDACLOPRIDO + BETA-CIFLUTRINA foi classes 3 e 2, para os hospedeiros A. kuehniella e $H$. zea, respectivamente (Tabela 2).

Por estes resultados pode-se constatar que baixas taxas de parasitismos, com maiores reduções do parasitismo,

Tabela 2: Percentuais de parasitismo, redução do parasitismo (RP), percentuais de emergência de T. pretiosum, em ovos dos hospedeiros A. kuehniella e H. zea, e classes de seletividade dos inseticidas

\begin{tabular}{|c|c|c|c|c|c|c|}
\hline \multirow{2}{*}{ Tratamentos } & \multicolumn{6}{|c|}{ Hospedeiros } \\
\hline & A. kuehniella & $\mathbf{R P}^{\mathbf{1}}$ & $\mathbf{C}^{2}$ & H. zea & $\mathbf{R P}^{1}$ & $\mathbf{C}^{2}$ \\
\hline IMIDACLOPRIDO + & $13,33 \mathrm{~dB}$ & 83,81 & 3 & $26,25 \mathrm{cA}$ & 67,48 & \\
\hline BETA-CIFLUTRINA & 2 & & & & & \\
\hline METHOMYL & $47,92 \mathrm{cA}$ & 41,80 & 2 & $41,50 \mathrm{bA}$ & 48,58 & 2 \\
\hline ACETAMIPRID & $62,50 \mathrm{bA}$ & 24,09 & 1 & $46,43 \mathrm{bB}$ & 42,48 & 2 \\
\hline SPINOSAD & $78,57 \mathrm{aA}$ & 4,57 & 1 & $85,67 \mathrm{aA}$ & $(6,14)$ & - \\
\hline TESTEMUNHA & $82,33 \mathrm{aA}$ & - & - & 80,71 aA & - & - \\
\hline $\mathrm{CV}(\%)$ & & & & 22,57 & & \\
\hline $\mathrm{F}$ & & & & 3,111 & & \\
\hline Glr & & & & 101 & & \\
\hline$p$ & & & & $<0,05$ & & \\
\hline
\end{tabular}

\begin{tabular}{|c|c|c|}
\hline \multicolumn{3}{|c|}{ Emergência $(\%)^{*}$} \\
\hline \multirow[t]{2}{*}{ Tratamentos } & \multicolumn{2}{|c|}{ Hospedeiros } \\
\hline & A. kuehniella & H. zea \\
\hline IMIDACLOPRIDO + BETA-CIFLUTRINA & $91,67 \mathrm{aA}$ & $100,00 \mathrm{aA}$ \\
\hline METHOMYL & $30,16 \mathrm{bB}$ & $98,33 \mathrm{aA}$ \\
\hline ACETAMIPRID & $89,41 \mathrm{aA}$ & $74,58 \mathrm{bA}$ \\
\hline SPINOSAD & $99,35 \mathrm{aA}$ & $100,00 \mathrm{aA}$ \\
\hline TESTEMUNHA & $97,10 \mathrm{aA}$ & $98,71 \mathrm{aA}$ \\
\hline $\mathrm{CV}(\%)$ & 20,75 & \\
\hline $\mathrm{F}$ & 20,947 & \\
\hline Glr & 101 & \\
\hline$p$ & $<0,01$ & \\
\hline
\end{tabular}


estão associadas aos inseticidas de elevado grau de toxicidade. Também se pode verificar que, dependendo do hospedeiro, essas taxas podem ter variações, as quais foram estatisticamente significativas, como ocorreu no tratamento com IMIDACLOPRIDO + BETA-CIFLUTRINAE ACETAMIPRID.

Produtos classificados como de elevado grau de toxicidade podem também expressar essas características para os inimigos naturais, conforme verificado em nossos estudos para Trichogramma. Esse comportamento também foi verificado por Scholz \& Zalucki (2000), que relataram que, quando ovos de Helicoverpa armigera (Hübner, 1805) (Lepidoptera: Noctuidae), tratados com METHOMYL, foram oferecidos à T. pretiosum, houve brusca redução da sobrevivência dos adultos. Avaliando IMIDACLOPRIDO e BETA-CIFLUTRINA isolados, Czepak et al. (2005) relatam redução para $60 \%$, na presença de inimigos naturais, após três e sete dias da aplicação, fato este que pode ser explicado pela elevada toxicidade desses produtos (Classe toxicológica II - Altamente tóxico) (Tabela 1). O que se pode constatar nesta pesquisa, e em uma gama delas, relacionadas com este assunto, é que os ingredientes ativos e suas concentrações devem ser considerados como os fatores primordiais da escolha de um inseticida para associá-lo a liberações de Trichogramma (Pratissoli, et al. 2009 e 2010b; Nörnberg, et al., 2011; Stefanello Júnior et al., 2012).

Para o parâmetro percentual de emergência dos descendentes de $T$. pretiosum, houve interação dos fatores Inseticidas e Hospedeiros ( $\mathrm{F}=20,947$; $\mathrm{glr}=107$; $\mathrm{p}<0,001)$ (Tabela 2). No entanto, na maioria dos tratamentos não foram percebidas alterações dos percentuais de emergência, quando, na maioria, foram superiores a $89 \%$, exceto quando esse parâmetro foi analisado no inseticida METHOMYL, quando este foi aplicado sobre o hospedeiro A. kuehniella, e, no ACETAMIPRID, quando aplicado sobre o hospedeiro $H$. zea $(30,16$ e 74,58\%), respectivamente. Também pôde ser observado que o inseticida METHOMYL reduziu a percentagem de emergência de ovos de A.kuehniella, quando comparados aos de H. zea (30,16 e $98,33 \%)$, respectivamente.

Com base nos resultados, independentemente da classe toxicológica dos produtos (Tabela 1), pode-se verificar que o hospedeiro influenciou a taxa de emergência e parasitismo de $T$. pretiosum. Essa influência pode estar relacionada com diferentes ações dos ingredientes ativos, no aspecto físico-químico, de acordo com as características do ovo da praga, ou seja, a influência varia de acordo com a espécie hospedeira, o que ocorreu, neste trabalho, para os parâmetros avaliados. Assim, as características dos ovos dos dois hospedeiros se diferenciam, pois os de $H$. zea apresentam forma hemisférica e saliências laterais, com 1 mm de diâmetro (Michereff Filho et al.,
2012) e, os de A. kuehniella, forma oval alongada, coloração branca, 0,58 mm de comprimento e $0,33 \mathrm{~mm}$ de largura e pesam em média $23 \mu \mathrm{g}$ (Brindley, 1930), podendo esta diferença entre as características dos ovos ter influenciado os resultados. .

De acordo com Pratissoli et al. (2004), os principais fatores do hospedeiro que podem interferir no parasitismo de Trichogramma são a área superficial do ovo, a espessura do córion, a presença de voláteis e sua qualidade nutricional. As características físico-químicas dos ovos dos insetos são peculiares a cada espécie, visto que cada inseto apresenta determinadas necessidades básicas para a sobrevivência, em seus habitats específicos. Desta forma, quanto mais heterogêneo o ambiente, maior será a estruturação físico-química dos ovos, para garantir a sobrevivência dos descendentes (Trougakos \& Margaritis, 2002). Ainda de acordo com Pratissoli et al. (2009), $T$. pretiosum tem um comportamento diferenciado em relação ao parasitismo, emergência dos ovos, número de indivíduos por ovo e longevidade dos descendentes, quando submetido a inseticidas de diferentes ingredientes ativos, em vários hospedeiros.

Outro fato que pode ter ocorrido é que a presença dos inseticidas na superfície dos ovos pode ter mascarado suas características físico-químicas, como observado por Bestete (2011), quando a aplicação de óleo de mamona em ovos de H. zea levou a uma influência no comportamento do parasitoide na aceitação do hospedeiro, com uma menor taxa de parasitismo.

Para o Experimento II (fungicidas), não houve interação entre os níveis dos fatores Fungicidas e Hospedeiro para o parâmetro parasitismo $(\mathrm{F}=0,115 ; \mathrm{glr}=101 ; \mathrm{p}=0,9515)$, bem como não houve efeito do fator Hospedeiro para este parâmetro $(F=0,049 ; g l r=101 ; p=0,8248)$. Por outro lado, houve efeito do fator Fungicida sobre o parasitoide ( $\mathrm{F}=$ 104,463; glr = 101; $\mathrm{p}<0,001)$. Todos os fungicidas afetaram significativamente o parasitismo de $T$. pretiosum (Tabela 3). No entanto, a menor taxa de redução foi verificada no protetor de plantas ACIBENZOLAR-S-METHYL (10,78 vezes), seguido de MACOZEB + OXICLORETO DE COBRE (48,45 vezes), MANCOZEB (61,51 vezes) e a maior redução do parasitismo foi observada para o fungicida DIMETOMORPH+MANCOZEB, o qual afetou drasticamente o parasitismo, com uma redução de 73,06 vezes, quando comparado com a das testemunhas.

Com base na classificação da IOBC, os tratamentos DIMETOMORPH + MANCOZEB, MACOZEB + OXICLORETO DE COBRE e MANCOZEB enquadraramse na classe 2 (levemente nocivos). O tratamento ACIBENZOLAR-S-METHYL, por sua vez, ficou na classe 1 (inócuo) (Tabela 3).

A redução do desempenho de Trichogramma, causada por MANCOZEB, já foi relatada por Pratissoli et al. 
(2010b) e Carvalho et al. (2012), que demonstraram que esse ingrediente ativo tem ação sobre os ovos dos hospedeiros. Nesta pesquisa, pôde-se constatar que todos os fungicidas, cuja formulação contém MANCOZEB ,apresentaram as maiores taxas de redução do parasitismo. A variação ocorrida entre os produtos pode estar associada à concentração do ingrediente MANCOZEB, visto que eles diferem quanto à classe toxicológica (Tabela 1). Apesar da menor concentração de MANCOZEB no fungicida DIMETOMORPH + MANCOZEB, pelos resultados, podese verificar que a associação com o DIMETOMORPH promoveu um sinergismo, o qual, possivelmente, intensificou a taxa de redução. Nesta pesquisa, a redução do parasitismo de $48,45 \%$, observada para MACOZEB + OXICLORETO DE COBRE, foi superior à relatada por Giolo et al. (2007) que não observaram redução do parasitismo de $T$. pretiosum em ovos de A. kuehniella.

Com relação ao parâmetro percentual de emergência, houve interação entre os fatores Fungicida e Hospedeiro $(\mathrm{F}=5,571 ; \mathrm{glr}=101 ; \mathrm{p}=0,0014)$, oscilando entre 92,26 e $100 \%$ de emergência (Tabela 3). Analisando-se os desdo- bramentos do fator Fungicidas dentro dos níveis do fator Hospedeiros, a aplicação com o fungicida MANCOZEB resultou em menor percentual de emergência dos descendentes de T. pretiosum para o hospedeiro A. kuehniella $(92,26 \%)$, enquanto para o hospedeiro $H$. zea a menor emergência ocorreu para o tratamento DIMETOMORPH + MANCOZEB (91,67\%). Ao analisar o desdobramento dos níveis do fator Hospedeiros dentro dos níveis do fator Fungicidas, somente não houve diferença entre os hospedeiros para o fungicida MANCOZEB + OXICLORETO DE COBRE. Em face disso, pode-se dizer que o fator hospedeiro foi o responsável pelas variações. Apesar da diferença observada, o percentual de emergência foi superior a $91 \%$ para todos os fungicidas testados, independentemente do hospedeiro utilizado. Da mesma forma, a ausência de reduções expressivas do percentual de emergência dos descendentes do parasitoide também foi reportada por outros autores (Carmo et al. 2010; Carvalho et al. 2012; Pratissoli et al. 2010b), o que indica que esta variável sofre pouca ou nenhuma influência de fungicidas.

Tabela 3: Percentuais de parasitismo, redução do parasitismo (RP), percentuais de emergência de T. pretiosum, em ovos dos hospedeiros A. kuehniella e H. zea, e classes de seletividade dos fungicidas

\begin{tabular}{lccc}
\hline Tratamentos & Parasitismo $(\boldsymbol{\%}) *$ & $\mathbf{R P}^{\mathbf{1}}$ & $\mathbf{C}^{\mathbf{2}}$ \\
\hline DIMETOMORPH+MANCOZEB & $22,65 \mathrm{e}$ & 73,06 & 2 \\
ACIBENZOLAR-S-METHYL & $75,00 \mathrm{~b}$ & 10,78 & 1 \\
MANCOZEB+OXICLORETO DE COBRE & $43,33 \mathrm{c}$ & 48,45 & 2 \\
MANCOZEB & $32,35 \mathrm{~d}$ & 61,51 & - \\
TESTEMUNHA A. kuehniella & $82,33 \mathrm{a}$ & - & - \\
TESTEMUNHA H. zea & $85,80 \mathrm{a}$ & - & - \\
TESTEMUNHA (MÉDIA) & $84,07-$ & & \\
CV $(\%)$ & 19,79 & \\
F & 104,463 & \\
Glr & 101 & & \\
$p$ & $<0,01$ & & \\
\hline
\end{tabular}

\begin{tabular}{|c|c|c|}
\hline \multicolumn{3}{|c|}{ Emergência $(\%)^{* *}$} \\
\hline \multirow{2}{*}{ Tratamentos } & \multicolumn{2}{|c|}{ Hospedeiros } \\
\hline & A. kuehniella & H. zea \\
\hline DIMETOMORPH+MANCOZEB & $100,00 \mathrm{aA}$ & $91,67 \mathrm{bB}$ \\
\hline ACIBENZOLAR-S-METHYL & $100,00 \mathrm{aA}$ & $97,68 \mathrm{aB}$ \\
\hline MANCOZEB+OXICLORETO DE COBRE & $100,00 \mathrm{aA}$ & $100,00 \mathrm{aA}$ \\
\hline MANCOZEB & $92,26 \mathrm{bB}$ & $98,44 \mathrm{aA}$ \\
\hline TESTEMUNHA & $97,10 \mathrm{aA}$ & $98,71 \mathrm{aA}$ \\
\hline $\mathrm{CV}(\%)$ & 5,49 & \\
\hline $\mathrm{F}$ & 5,571 & \\
\hline Glr & 101 & \\
\hline$p$ & $<0,01$ & \\
\hline \multicolumn{3}{|c|}{ * Médias seguidas de mesma letra minúsculas na coluna não diferem entre si pelo teste de Tukey $(p<0,05)$; } \\
\hline
\end{tabular}


Assim, com os presentes dados, tanto inseticidas quanto fungicidas mais seletivos devem ser utilizados, quando há conjugação da utilização de agrotóxicos com liberação de Trichogramma, sendo que é de grande importância intercalar e respeitar os intervalos entre a liberação e a aplicação desses produtos, de forma que assegure o parasitismo, sendo este um parâmetro muito afetado. De acordo com Pinto (2005), o ideal seria fazer a liberação dos parasitoides e somente após três dias a pulverização com agrotóxicos, sendo que, dessa forma, as fêmeas conseguiriam atuar com mais eficiência.

\section{CONCLUSÕES}

Os inseticidas, os fungicidas e o hospedeiro influenciaram no parasitismo de T. pretiosum;

As maiores reduções do grau de parasitismo estão associadas aos inseticidas de elevado grau de toxicidade;

O inseticida SPINOSAD foi o que ocasionou os maiores percentuais de parasitismo de $T$. pretiosum enquanto que IMIDACLOPRIDO+BETA-CIFLUTRINA proporcionou os menores, para ambos os hospedeiros;

Somente o tratamento SPINOSAD enquadrou-se na classe 1 (inócuo), na classificação da IOBC, para ambos os hospedeiros e ACETAMIPRID foi classe 1 para o hospedeiro A. kuehniella;

O efeito sobre a emergência foi observado apenas para o METHOMYL, quando em A. kuehniella, e ACETAMIPRID, em H. zea;

Todos os fungicidas afetaram significativamente o parasitismo de T. pretiosum;

A menor taxa de redução de parasitismo foi observada para o fungicida ACIBENZOLAR-S-METHYLe, a maior, para DIMETOMORPH+MANCOZEB, obtendo classes 1 (inócuo) e 2, respectivamente, com base na classificação da IOBC:

Não houve reduções expressivas do percentual de emergência dos descendentes quando os fungicidas foram aplicados.

\section{FINANCIAMENTO E APOIO}

Nossos sinceros agradecimentos à FAPES e ao CNPq, pelo apoio financeiro.

\section{REFERÊNCIAS}

Agrofit (2015) Banco de informações sobre os produtos agrotóxicos e afins registrados no Ministério da Agricultura. Disponível em: <http://www.agricultura.gov.br/servicos-e-sistemas/sistemas/agrofit $>$. Acessado em: 24 de setembro de 2015.

Almeida GD, Zanuncio JC, Pratissoli D, Andrade GS, Cecon PR \& Serrão JE (2010) Effect of azadirachtin on the control of Anticarsia gemmatalisand its impact on Trichogramma pretiosum. Phytoparasitica, 38:413-419.
Alves FR, Jesus Junior WC, Pratissoli D, Polanczyk RA, Zanuncio Junior JS, Holtz AM \& Vianna UR (2007) Manejo fitossanitário de doenças e pragas: novas perspectivas. In: Jesus Junior WC, Polanczyk RA, Pratissoli D, Pezzopane JEM \& Santiago T (Eds.) Atualidades em defesa fitossanitária. Alegre, UFES. p.383416.

Bestete LR (2011) Produtos alternativos e associação com Trichogramma pretiosum Riley (Hym.: Trichogrammatidae) visando o controle de Helicoverpa zea (Boddie) (Lep.: Noctuidae) em tomateiro. Dissertação de Mestrado. Universidade Federal de Pernambuco, Pernambuco. 54p.

Brindley TA (1930) The growth and development of Ephestia kuehniella Zeller (Lepidoptera) and Tribolium confusum Duval (Coleoptera) under controlled conditions of temperature and relative humidity. Annals of Entomological Society of America, 23:741-757.

Carmo EL do, Bueno AF, Bueno RCOF, Vieira SS, Goulart MMP \& Carneiro TR (2010) Seletividade de produtos fitossanitários utilizados na cultura da soja para pupas de Trichogramma pretiosum Riley, 1879 (Hymenoptera: Trichogrammatidae). Arquivos do Instituto Biológico, 77:283-290.

Carvalho JR, Pratissoli D, Paes JPP, Zuim V \& Stinguel P (2012) Seletividade de fungicidas utilizados na cultura do tomateiro (Lycopersicum esculentum, MILL.) a Trichogramma pretiosum. Nucleus, 9:01-08.

Czepak C, Fernandes PM, Albernaz KC, Rodrigues OD, Silva LM, Silva EA, Takatsuka FT \& Borges JD (2005) Seletividade de inseticidas ao complexo de inimigos naturais na cultura do algodão (Gossypium hirsutum). Pesquisa Agropecuária Tropical, $35: 123-127$.

Ferreira DF (2003) Sisvar. Versão 5.1. Disponível em: <http:// www.dex.ufla.br/danielff/prog.htm>. Acessado em: 01 de junho de 015 .

Ferreira DF (2011) Sisvar: a computer statistical analysis system. Ciência e Agrotecnologia, 35:1039-1042.

Giolo FP, Grützmacher AD, Manzoni CG, Lima CAB \& Nörnberg SD (2007) Toxicidade de produtos fitossanitários utilizados na cultura do pessegueiro sobre adultos de Trichogramma pretiosum. Bragantia, 66:423-431.

Greene GL, Lepla NC \& Dickerson WA (1976) Velvetbean caterpillar: a rearing procedure and artificial medium. Journal of Economic Entomology, 69:488-497.

Hassan SA, Halsall N, Gray AP, Kuehner C, Moll M, Bakker FM, Roembke J, Yousef A, Nasr F \& Abdelgader H (2000) A laboratory method to evaluate the side effects of plant protection products on Trichogramma cacoeciae Marchal (Hym., Trichogrammatidae). In: Candolfi MP, Blümel S, Forster R, Bakker FM, Grimm C, Hassan SA, Heimbach U, Mead-Briggs MA, Reber B, Schmuck R \& Vogt H (Eds.) Guidelines to evaluate side-effects of plant protection products to non-target arthropods. Gent, IOBC/ WPRS. p.107-119.

Hassan SA, Hafes H, Degrande PE \& Herai H (1998) The side effects of pesticides on the egg parasitoid Trichogramma caecoeciae Marchal (Hym., Trichogrammatidae), acute doseresponse and persistence tests. Journal of Applied Entomology, 122:569-573.

Luz JMQ, Shinzato AV \& Silva MAD (2007) Comparação dos sistemas de produção de tomate convencional e orgânico em cultivo protegido. Bioscience Journal, 23:07-15.

Michereff Filho M, Moura AP de, Guimarães JA, Reyes CP, Carvalho ADF de, Amaro GB, Lopes JF \& Liz RS de (2012) Recomendações técnicas para o controle de pragas do pepino. Brasília, Embrapa. 15p. (Circular Técnica, 109). 
Nörnberg SD, Grützmacher AD, Kovaleski A, Finatto JA \& Paschoal MDF (2011) Persistência de agrotóxicos utilizados na produção integrada de maçã a Trichogramma pretiosum. Ciência e Agrotecnologia, 35:305-313.

Parra JRP (2001) Técnicas de criação de insetos para programas de controle biológico. Piracicaba, ESALQ. 134p.

Pinto A de S (2005) A cultura do tomate no Brasil exagera no uso de agrotóxicos e o controle biológico de pragas é viável e um importante substituto para os agricultores conscientes. Disponível em: <http://gebio.com.br/site/campo/controle-biologicode-pragas-do-tomateiro/>. Acessado em: 23 de setembro de 2015.

Pratissoli D, Dalvi LP, Polanczyk RA, Andrade GS, Holtz AM \& Nicoline HO (2010a) Características biológicas de Trichogramma exiguum em ovos de Anagasta kuehniella e Sitotroga cerealella. Idésia, 28:39-42.

Pratissoli D, Milanez AM, Barbosa WF, Celestino FN, Andrade GS \& Polanczyk RA (2010b) Side effects of fungicides used in cucurbitaceous crop on Trichogramma atopovirilia Oatman \& Platner (Hymenoptera: Trichogramatidae). Chilean Journal of Agricultural Research, 70:323-327.

Pratissoli D, Vianna UR, Furtado GO, Zanuncio JC, Polanczyk RA, Barbosa WF \& Carvalho JR (2009) Seletividade de inseticidas a Trichogramma pretiosum Riley (Hymenoptera: Trichogrammatidae) em diferentes hospedeiros. Boletín de Sanidad Vegetal Plagas, 35:347-353.
Pratissoli D, Polanczyk RA, Dalvi LP, Silva AF da, Melo DF \& Celestino FN (2007) Primeiro Relato de Trichogramma bruni Nagaraja (Hymenoptera: Trichogrammatidae) Parasitando Ovos de Urbanus proteus (L.) (Lepidoptera: Hesperiidae) em Feijão-Vagem. Neotropical Entomology, 36:487-488.

Pratissoli D, Holtz AM, Gonçalves JR, Oliveira RC de \& Vianna UR (2004) Características biológicas de linhagens de Trichogramma pretiosum, criados em ovos de Sitotroga cerealella e Anagasta kuehniella. Horticultura Brasileira, 22:562-565

Pratissoli D, Fornazier MJ, Holtz AM, Gonçalves JR, Chioramital AB \& Zago H (2002) Ocorrência de Trichogramma pretiosum em áreas comerciais de tomate, no Espirito Santo, em regiões de diferentes altitudes. Horticultura Brasileira, 21:73-76.

Scholz BCG \& Zalucki MP (2000) The effects of two new insecticides on the survival of adult Trichogramma pretiosum Riley in sweet corn. In: Austin A \& Dowton M (Eds.) Hymenoptera: Evolution, Biodiversity and Biological Control. Canberra, CSIRO Publishing. p.381-387.

Stefanello Júnior GJ, Grutzmacher AD, Spagnol D, Pasini RA, Bonez C \& Moreira DC (2012) Persistência de agrotóxicos utilizados na cultura do milho ao parasitoide Trichogramma pretiosum Riley, 1879 (Hymenoptera: Trichogrammatidae). Ciência Rural, 42:01-06.

Trougakos I \& Margaritis L (2002) Novel morphological and Physiological Aspects of insect eggs. In: Hilker M \& Meiners $\mathrm{T}$ (Eds.) Chemoecology of insect eggs and egg deposition. Berlin, Blackwell Publishing. p.03-36. 